\title{
Papel de la ecoendoscopia en la evaluación de la dispepsia no investigada en una población colombiana
}

\section{The role of endoscopic ultrasound in evaluating patients with dyspepsia in a Colombian population}

\author{
Martín A. Gómez Zuleta, ${ }^{1 *}$ William Otero Regino, ${ }^{2}$ Óscar Ruíz Morales. ${ }^{3}$
}

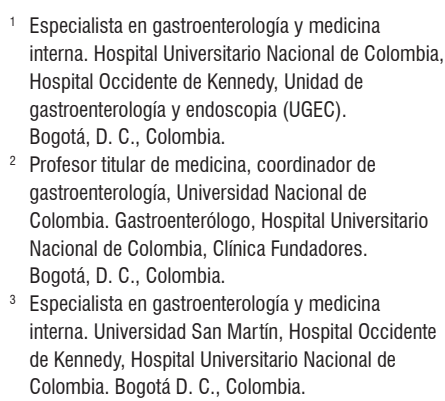

2 Profesor titular de medicina, coordinador de gastroenterología, Universidad Nacional de Colombia. Gastroenterólogo, Hospital Universitario Nacional de Colombia, Clínica Fundadores. Bogotá, D. C., Colombia.

3 Especialista en gastroenterología y medicina interna. Universidad San Martín, Hospital Occidente de Kennedy, Hospital Universitario Nacional de Colombia. Bogotá D. C., Colombia.

*Correspondencia: martinalonsogomezz@gmail.com.

Fecha recibido: $25 / 03 / 18$

Fecha aceptado: 29/08/18

\begin{abstract}
Resumen
La dispepsia se define como un dolor o molestia abdominal superior que se considera originado en el tracto gastrointestinal superior. Muchas enfermedades y condiciones clínicas pueden producir dispepsia como la úlcera péptica, cáncer gástrico o esofágico, medicamentos, litiasis biliar, pancreatitis, cáncer de páncreas, entre otras. Por una parte, tradicionalmente la dispepsia solo se evalúa con la endoscopia digestiva y su producción solo es del $27 \%$. Por otra parte, la ecoendoscopia (ECE), al combinar una imagen endoscópica y una imagen ecográfica, podría ampliar el rango diagnóstico al detectar más causas de dispepsia que puedan ayudar a tratar al paciente de manera más oportuna. Objetivo: evaluar si la ECE aumenta el rendimiento diagnóstico que tiene la endoscopia ( $27 \%$ en nuestro medio) en el enfoque inicial de la dispepsia no investigada (DPNI). Materiales y métodos: estudio prospectivo, de prevalencia analítica en pacientes adultos con DPNI, que consultaron a una institución universitaria de Colombia. Los pacientes incluidos consultaron a la unidad de gastroenterología durante el período comprendido entre enero a octubre del 2016 y se programaron para endoscopia digestiva alta (EDA). Bajo sedación guiada por anestesiólogo primero se realizó la fase endoscópica evaluando el esófago, estómago y duodeno; después se realizó la fase ecográfica y se inició el examen de manera retrógrada evaluándose el páncreas en su totalidad, la vía biliar extrahepática, la vesícula biliar, el tronco celíaco, el lóbulo izquierdo del hígado y la región mediastinal. Todas las anormalidades se anotaban en el formulario de ingreso del paciente. Resultados: En total se incluyeron a 60 pacientes, de los cuales el $65 \%$ son de sexo femenino con un promedio de edad de 40,8 (desviación estándar: 12,5). Los hallazgos en la fase endoscópica de la ECE fueron principalmente gastritis crónica en 43 pacientes $(71,6$ $\%)$, el resto tenía una lesión estructural (17 pacientes): esofagitis en $5(8,3 \%)$, úlcera gástrica en $2(3,3 \%)$, úlcera duodenal en $5(8,3 \%)$, cáncer gástrico en $4(6,6 \%)$ y lesión subepitelial gástrica (GIST) en $1(1,6 \%)$. Los hallazgos en la fase ecográfica de la ECE fueron colelitiasis en $11(18,3 \%)$, coledocolitiasis en $1(1,6$ $\%$ ) y pancreatitis crónica en 5 (8,3\%). Aunque solo el 28,3 \% (17) de los pacientes con dispepsia tuvo un hallazgo estructural en la fase endoscópica, 18 pacientes (30\%) presentaron algún hallazgo positivo en la fase ecográfica, es decir que el rendimiento subió a un 58,3\% $(p<0,001)$. Conclusión: este estudio, aunque pequeño, sugiere que la ECE como método inicial de enfoque en la evaluación de la dispepsia podría llegar a ser útil dado que aumenta el rendimiento diagnóstico en este grupo de pacientes, pasando de un $28,3 \%$ a $58,3 \%$, lo cual es muy significativo si se tiene en cuenta que estos pacientes con dispepsia y endoscopia negativa usualmente se catalogan como funcionales y solo se les formula medicamentos. Sin embargo, se deben reconocer las fallas metodológicas del estudio y consideramos que es una exploración inicial y deben plantearse estudios más grandes y controlados para confirmar este trabajo; además, se debe tener en cuenta el costo del examen, que es mucho mayor que el de la EDA.
\end{abstract}

Palabras clave

Ecoendoscopia, evaluación, dispepsia, cáncer gástrico. 


\begin{abstract}
Dyspepsia is defined as upper abdominal pain or discomfort that is considered to originate in the upper gastrointestinal tract. Many diseases and clinical conditions can cause dyspepsia. Among others, they include peptic ulcers, gastric and esophageal cancer, medications, biliary lithiasis, pancreatitis, and pancreatic cancer. Traditionally, dyspepsia is only evaluated with digestive endoscopy whose diagnostic yield is only $27 \%$. On the other hand, endoscopic ultrasound combines an endoscopic image and an ultrasound image thereby potentially broadening diagnostic range to detect more of the causes of dyspepsia allowing treatment of patients in a timelier manner. Objective: To evaluate whether endoscopic ultrasound increases the diagnostic yield of endoscopy ( $27 \%$ in our environment) in the initial approach to previously unstudied dyspepsia. Materials and methods: This is a prospective study of analytical prevalence in adult patients with previously unstudied dyspepsia who were examined at a university institution in Colombia. The patients included were seen in the gastroenterology unit from January to October 2016 and underwent upper digestive endoscopy and endoscopic ultrasound. Under anesthesiologist-guided sedation, the stomach and duodenal esophagus were first evaluated endoscopically. Then retrograde endoscopic ultrasound was used to evaluate the pancreas in its entirety, the extra hepatic bile duct, the gallbladder, the celiac trunk, the left lobe of the liver and the mediastinal region. All abnormalities were noted on the patient's admission form. Results: In total we included 60 patients of whom $65 \%$ were female and whose average age of was 40.8 years (SD: 12.5$)$. The findings in the endoscopic phase of the endoscopic ultrasound were mainly chronic Gastritis 43 patients $(71.6 \%)$, the rest had a structural lesion (17 patients): esophagitis $5(8.3 \%)$, gastric ulcer $2(3.3 \%)$, duodenal ulcer $5(8.3 \%)$, gastric cancer, $4(6.6 \%)$, gastric subepithelial lesion (GIST) $1(1.6 \%)$. In the endoscopy phase, we found 11 cases of cholelithiasis $(18.3 \%)$, one case of choledocholithiasis $(1.6 \%)$, and five cases of chronic pancreatitis $(8.3 \%)$. Only 17 patients of these patients $(28.3 \%)$ had a structural finding in the endoscopy phase, but 18 additional patients $(30 \%)$ had some positive finding in the ultrasound phase. In other words, the diagnostic yield rose to $58.3 \%(p<0.001)$. Conclusion: Although this study's sample size is small, it suggests that using endoscopic ultrasound in the initial evaluation of dyspepsia could be useful since it increased diagnostic yield in this group of patients from 28.3 to $58.3 \%$. This is very significant because patients with dyspepsia and negative endoscopy are usually classified as functional and only treated with medications. However, in recognition of the methodological limitations of this study, it should be considered an initial exploration. Larger, controlled studies should be considered to confirm this work. Another factor that should be considered is the cost of endoscopic ultrasound which is much higher than the upper digestive endoscopy.
\end{abstract}

Keywords

Endoscopic ultrasound, evaluation, dyspepsia, gastric cáncer.

\section{INTRODUCCIÓN}

La dispepsia es un síndrome clínico caracterizado por dolor o malestar en el abdomen superior (1). Afecta por lo menos al $20 \%$ de la población mundial (2) y tiene múltiples causas incluidas patologías benignas y malignas $(3,4)$. De manera incorrecta, con frecuencia, se reemplaza por las denominaciones gastritis crónica o enfermedad acidopéptica. En algunos países se ha encontrado que la incidencia anual de la dispepsia es aproximadamente del $1 \%$ y se calcula que una de cada dos personas consultará en algún momento de su vida por síntomas dispépticos. Representa el $5 \%$ de la consulta en medicina general y aproximadamente del $20 \%$ al $30 \%$ de la consulta en gastroenterología (5-7) y afecta negativamente la calidad de vida (8). Cuando un paciente tiene dispepsia y no se le ha estudiado su causa, se denomina dispepsia no investigada (DPNI) (8). El enfoque de los pacientes adultos incluye la endoscopia digestiva alta (EDA) y, ocasionalmente, ecografía abdominal superior (EAS) $(1,3)$. Con base en los resultados, la dispepsia puede ser secundaria u orgánica si hay alteraciones evidentes, o dispepsia funcional (DPF) si el examen no muestra alteraciones que expliquen los síntomas $(2,3)$.

La utilización temprana de la endoscopia es costo-efectiva $(9,10)$. El momento de la EDA varía entre los diferentes lugares. En los países desarrollados, se recomienda después de los 55 años y en los que están en vías de desarrollo con alta incidencia de cáncer gástrico, a los 35 años (3). En Colombia no se han realizado estudios de costo-efectividad en esta entidad, pero sí estudios de prevalencia de lesiones endoscópicas en pacientes con DPNI. Hace más de 10 años en un estudio prospectivo de pacientes con DPNI se encontró que el 73 \% tenía DPF (11). Otra investigación colombiana en pacientes con DPNI encontró colelitiasis en el $21 \%$ (12).

Globalmente, el rendimiento de la EDA como único método para identificar causas orgánicas no supera el $30 \%$ $(1,11)$. Por lo anterior, es necesario utilizar otros métodos que tengan mayor sensibilidad ya que, conforme se profundiza en el estudio de esos pacientes, se encuentran causas 
desconocidas hasta entonces. $\mathrm{Al}$ respecto, recientemente se ha descrito la dispepsia secundaria a Helicobacter pylori (13), una nueva entidad que en el pasado se desconocía y estaba involucrada dentro de la categoría de DPF. Actualmente se ha demostrado que aproximadamente el $5 \%$ de los pacientes con $H$. pylori se puede curar de la dispepsia si se erradica la infección $(13,14)$. Este nuevo hallazgo demuestra la necesidad de seguir investigando etiologías desconocidas que podrían explicar los síntomas dispépticos. Con el enfoque diagnóstico tradicional cuando la EDA es negativa, el diagnóstico es DPF y si no se realizan más estudios se trata al paciente con inhibidores de la bomba de protones (IBP), procinéticos o antidepresivos, entre otros (8), durante varios meses, lo cual puede enmascarar patologías no identificadas con probables complicaciones por las mismas.

La ecoendoscopia (ECE) es un examen que combina la imagen de una EDA convencional con la imagen ecográfica $y$, cuando se utiliza el equipo radial, la imagen ecográfica evalúa un rango de alrededor de $6 \mathrm{~cm}$ de todo el esófago, estómago y duodeno (15). Dado que el ecoendoscopio radial Pentax es frontal (diferente al equipo de Olympus, que es oblicuo), se puede hacer una endoscopia idéntica a una endoscopia convencional; luego se puede hacer la fase ecográfica. Esta visibilidad, más allá del lumen gastrointestinal superior, permitiría diagnosticar más patologías que pudieran asociarse con la dispepsia. Teniendo en cuenta la alta frecuencia de la dispepsia, así como la falta de investigaciones en nuestro medio diferentes a la EDA sobre otras etiologías de esta, se decide realizar el presente trabajo utilizando la ECE dentro del estudio de los pacientes con DPNI con el objetivo de evaluar si este examen aumenta el rendimiento diagnóstico de $27 \%$ que tiene la endoscopia en nuestro medio.

\section{MATERIALES Y MÉTODOS}

Estudio prospectivo de prevalencia analítica en pacientes adultos con DPNI, que consultaron a una institución universitaria de Colombia. Los pacientes incluidos habían consultado a la unidad de gastroenterología durante el período comprendido entre enero a octubre del 2016 y se programaron para EDA. Con la aceptación del paciente y previa firma de consentimiento, en vez de introducir el endoscopio estándar se utilizó el ecoendoscopio Pentax. La dispepsia se definió con base en los criterios de ROMA IV, la cual define la dispepsia como la presencia de síntomas superiores de plenitud posprandial, saciedad temprana y dolor o quemazón epigástrica. Si no hay lesión estructural en la endoscopia se define como dispepsia funcional (DPF) (8). Las indicaciones de endoscopia se tomaron de acuerdo con la guía de dispepsia de la Asociación Colombiana de Gastroenterología (ACG), que recomienda realizar la EDA a todos los pacientes mayores 35 años con DPNI, independientemente de tener o no síntomas de alarma (16). Se excluyeron del estudio a los pacientes que tuvieran contraindicación para realizar una endoscopia con sedación, pacientes con EDA previa, pacientes con antecedentes de cirugías gastrointestinales previas (incluidas las colecistectomías) y pacientes a quienes se les hubiera realizado colangiopancreatografía retrógrada endoscópica (CPRE) o estudios de imágenes del abdomen tales como ecografías, resonancia magnética (RM) o tomografía axial computada (TAC). La ECE se realizó en la forma usual (17).

Se describe brevemente el procedimiento: bajo sedación administrada por un anestesiólogo, se realizó una ECE radial con un ecoendoscopio marca Pentax, que a diferencia del equipo Olympus, tiene una visión frontal que permite hacer una endoscopia idéntica a un endoscopio convencional. Primero se realizó la fase endoscópica en la que se evaluó el esófago, estómago y duodeno, de acuerdo con el protocolo descrito por Yao (18), que consiste básicamente en la evaluación de 21 zonas en el tercio proximal, medio y distal del estómago en forma directa y en retrovisión. A los pacientes se les tomó dos biopsias del antro y dos del cuerpo para evaluar la infección por H. pylori, y biopsias adicionales si tenían lesiones estructurales aparentes. Después se realizó la fase ecográfica y el ecoendoscopio se llevó hasta la papila mayor, se inició el examen de manera retrógrada y se evaluó el páncreas en su totalidad, la vía biliar extrahepática, la vesícula biliar, el tronco celíaco, el lóbulo izquierdo del hígado y la región mediastinal. Todas las anormalidades se anotaban en el formulario de ingreso del paciente. El protocolo e investigación y el consentimiento informado los aprobó el comité de ética de la institución participante.

\section{RESULTADOS}

En total se incluyeron a 60 pacientes con edad promedio de 40,8 años $\pm 12,5$ años, $65 \%$ mujeres. Los hallazgos en la fase endoscópica de la ECE fueron principalmente gastritis crónica en $43 / 60$ pacientes $(71,6 \%)$ y lesiones estructurales en $17 / 60$, distribuidas delsiguientemodo: esofagitis en $5(8,3 \%)$, úlcera gástrica en $2(3,3 \%)$, úlcera duodenal en $5(8,3 \%)$, cáncer gástrico comprobado histológicamente en 4 (6,6\%) y lesión subepitelial gástrica (GIST) en 1 (1,6\%). El $70 \%$ (42/60), tenía H. pylori. En 8 pacientes (13\%) se encontró gastritis atrófica y en $6(10 \%)$ metaplasia intestinal.

Los hallazgos en la fase ecográfica de la ECE fueron colelitiasis en 11/60 (18,3\%), coledocolitiasis en $1(1,6 \%)$, pancreatitis crónica en $5 / 60(8,3 \%)$ por los criterios de Rosemont (19), quiste de páncreas (sugestivo de cistadenoma mucinoso) en 1 ( $1,6 \%)$, cáncer de páncreas en 1 (1,6 $\%$; el cual era estadio T2) y adenopatías mediastinales en 1 ( $1,6 \%$; que tenía también cáncer gástrico). Globalmente, 
$17 / 60$ pacientes $(28,3 \%)$ tuvieron alteraciones estructurales en la fase endoscopia y 18/60 (30\%) presentaron algún hallazgo positivo en la fase ecográfica, no vista en la fase endoscópica, es decir que los casos en los cuales se encontró un hallazgo en la ECE no tenían ningún hallazgo estructural en la endoscopia y técnicamente se considerarían como pacientes con DPF. Solo 25 pacientes $(41,6 \%)$ no tuvieron ningún hallazgo en la fase de la endoscopia y en la fase de la ECE. En todos los casos se logró visualizar adecuadamente la vesícula, la vía biliar, la cabeza, el cuerpo y la cola del páncreas. Los hallazgos globales se muestran en la Figura 1.

\section{DISCUSIÓN}

En el presente trabajo se encontró que en los pacientes con DPNI había alteraciones durante la fase endoscópica en $28,3 \%$ de ellos, hallazgo que es similar al $27 \%$ encontrado en una investigación previa de la Universidad Nacional (11), lo cual corrobora el poco rendimiento de la EDA en nuestro medio. En la fase ecográfica de la ECE, se detectaron en $30 \%$ de los pacientes lesiones adicionales que podrían explicar sus síntomas dispépticos, con lo cual el rendimiento final de este último procedimiento y con este equipo de ECE con visión frontal es de 58,3\% ( $p<0,001)$, es decir que solo $25(41,6 \%)$ de los 60 pacientes evaluados tuvieron un resultado normal.
El hallazgo de colelitiasis en el $18 \%$ de los pacientes coincide con la prevalencia de esta patología en occidente $(20$, 21). Sin embargo, no se puede concluir que la presencia de cálculos en la vesícula sea la causa de los síntomas dispépticos, ya que el síntoma más frecuentemente asociado con estos es el cólico. Para determinar la asociación de las dos entidades se necesitaría un estudio con mayor número de pacientes, probablemente de casos y controles. Sin embargo, todos los gastroenterólogos saben que los pacientes con colelitiasis no necesariamente se manifiestan con dolor cólico en el hipocondrio derecho (típico), sino que pueden manifestarse con síntomas en el epigastrio generalmente posprandiales, muy sugestivos de dispepsia. Si se sustrae el hallazgo de colelitiasis, la ECE supera la EDA en $12 \%$ y, dado el tipo de patologías pancreáticas encontradas, se puede inferir que, si en los pacientes con DNI solo se tiene en cuenta la EDA, cuando esta es negativa, erróneamente se concluiría que es DPF y el manejo sería con procinéticos, IBP o antidepresivos (22) de manera prolongada, que al no lograr mejoría significativa, podrían generar ansiedad en los pacientes y, para la seguridad de pacientes y médicos, en muchos casos la investigación continuaría con exámenes adicionales como ecografía abdominal, TAC u otros estudios $(8,22)$.

El hallazgo de patología pancreática en más del $11 \%$ y en un paciente con coledocolitiasis plantea un nuevo enfoque para los pacientes con DPF y síntomas persistentes. Ante

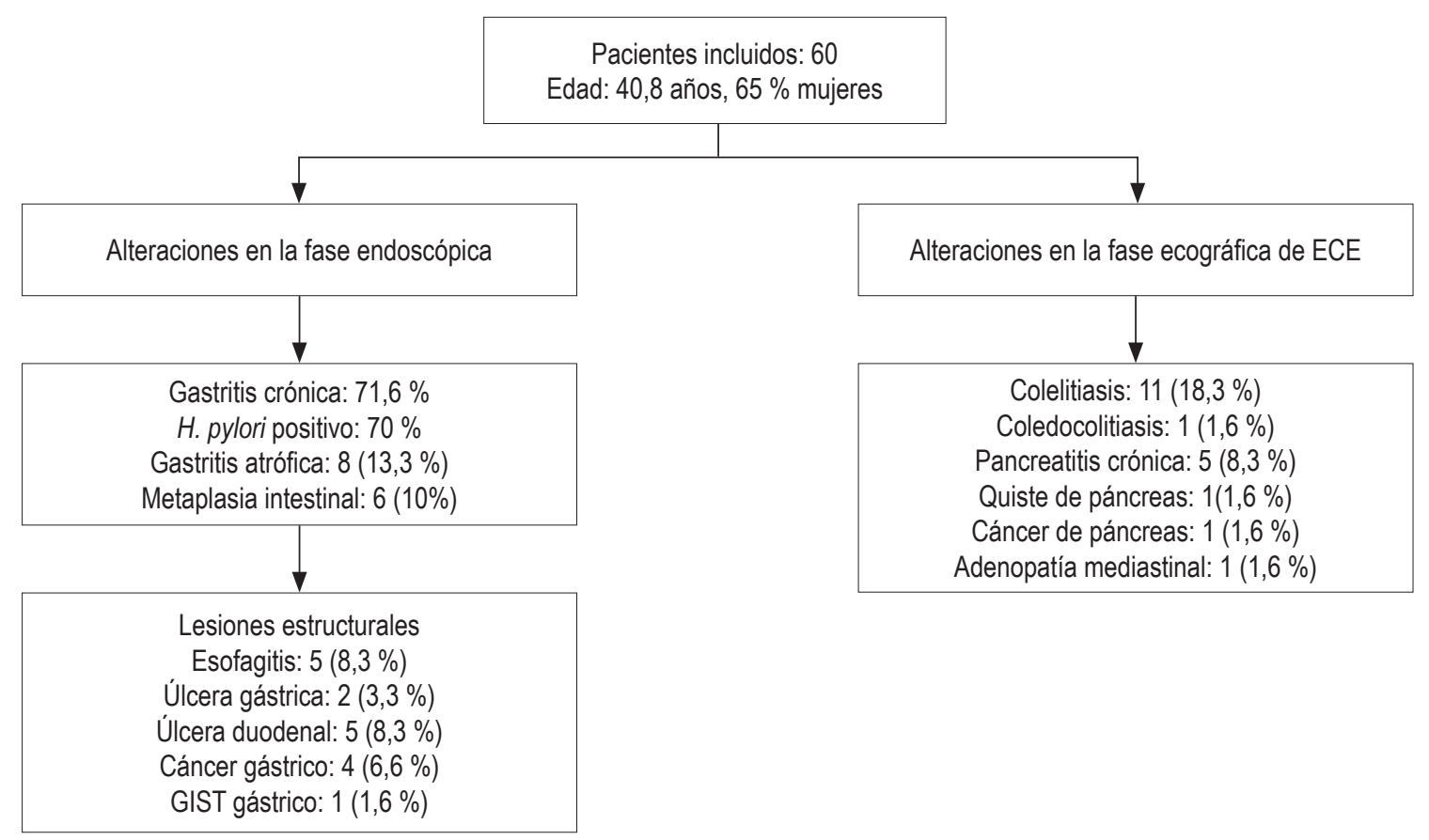

Figura 1. Resultados globales del estudio. 
ese tipo de pacientes, el próximo paso no sería repetir la EDA, sino una ECE biliopancreática e, incluso, hacer este examen en vez de ecografía, TAC o RM abdominal. En un estudio de hace más de 15 años se sugirió que la ECE podía ser más costo-efectiva (23). En un estudio más reciente, Chang y colaboradores (24) demostraron que en Estados Unidos la ECE como primer examen es más costo-efectiva que una EDA, seguida de ecografía abdominal ante la persistencia de los síntomas. Si se hiciera TAC abdominal o RM, serían más evidentes las ventajas de la ECE. Teniendo en cuenta los resultados de los estudios internacionales y los del presente estudio, consideramos que en nuestro medio es necesario y oportuno determinar el lugar de la ECE biliopancreática en los pacientes con DPNI. Por lo anterior se considera que, dado el alto costo de la ECE en nuestro medio, sería prematuro recomendar este examen en todos los pacientes con DPNI. Sin embargo, la prevalencia de patología biliopancreática demuestra que por lo menos sea el examen de elección ante un paciente con DPF que no mejore con los tratamientos convencionales.

Un aspecto muy importante que por el momento limitaría el uso de ECE como examen de rutina, por lo menos en nuestro medio, es que se ha exagerado y se sigue exagerando la complejidad de este procedimiento. Realizar una ECE gastroesofágica o del páncreas es un procedimiento relativamente sencillo que no toma más de 5 o 10 minutos y cuyo entrenamiento no es muy complejo. Esto recuerda lo que sucedía con la CPRE en sus inicios, hace más de 50 años, cuando se le consideraba un procedimiento muy complejo y solo realizable por muy pocos gastroenterólogos y también en muy pocos centros. Actualmente es un procedimiento de rutina realizado por muchos gastroenterólogos a nivel global sin tener en cuenta que la CPRE es un examen mucho más complejo y peligroso que una ECE (24). La complejidad real de la ECE es en la parte terapéutica o invasiva de la misma, tal como la estatificación del cáncer de páncreas, la punción o los drenajes. Por tanto, es momento de desmitificar la ECE no terapéutica y, entendiendo esa realidad, no debería ser difícil imaginar que en un futuro próximo se pudiera utilizar para la evaluación inicial de la DPNI, una patología compleja que curiosamente muchos consideran un problema insignificante o fácil, cuando se le denomina gastritis o enfermedad acidopéptica. En realidad, es un síndrome complejo de abordar, por su naturaleza recurrente, que adicionalmente deteriora la calidad de vida de los pacientes, gasta muchos recursos económicos de los sistemas de salud del mundo y adicionalmente podría tener etiologías serias y no sospechadas, como las encontradas en el presente estudio.

Hasta donde hemos revisado, para nuestro conocimiento este es el primer estudio que se hace en América Latina sobre ECE con equipo frontal en la evaluación inicial de los pacientes con dispepsia. Un estudio de diseño similar, de hace más de 15 años (25) no detectaba todas las lesiones endoluminales cuando se comparaba con la endoscopia ya que se usó un ecoendoscopio marca Olympus, que tiene solo visión oblicua, que a diferencia de las otras marcas (Fuji o Pentax) que tienen visión frontal, no permite hacer una endoscopia convencional (15) porque limita la fase endoscópica, por lo que no se pudieron evaluar muchas áreas y esto puede contribuir a que permanecieran ocultas lesiones como úlceras o tumores.

Adicionalmente, es importante tener en cuenta que actualmente con frecuencia se ejerce una medicina defensiva (26), en la cual muchos pacientes presionan al médico para que se llegue a un diagnóstico rápido de su enfermedad porque están ansiosos con la misma y no aceptan que su dolor solo se explique por una gastritis y el médico se deja influenciar pidiendo múltiples exámenes que incluso pueden ser más costosos que una ECE.

Las limitaciones de esta investigación incluyen el tamaño de la muestra (no calculado) y que no es multicéntrico, por lo cual sería conveniente plantear estudios con una muestra de mayor tamaño para confirmar los hallazgos de este trabajo.

\section{CONCLUSIÓN}

En el presente estudio se encontró que en los pacientes con DPNI, la ECE supera el rendimiento de la EDA en $30 \%$, logrando identificar patologías en $58,3 \%$ frente a $28,3 \%$ (solo de la fase endoscópica) de los pacientes $(p<0,001)$. Cuando un paciente con DPNI tiene EDA negativa, con base en las recomendaciones actuales, tendría DPF y la conducta es la utilización indefinida de medicamentos cuya eficacia es moderada. Con los hallazgos del presente estudio, se plantea la posibilidad de que la ECE sea el examen inicial de evaluación o que, ante la persistencia de los síntomas, el próximo examen sería una ECE y no una ecografía o TAC o RM. Se justifica realizar estudios de costo-efectividad en nuestro medio para determinar si este examen podría ser el de primera elección por encima de la endoscopia convencional.

\section{Conflicto de interés}

Ninguno.

\section{REFERENCIAS}

1. Graham DY, Rugge M. Clinical practice: diagnosis and evaluation of dyspepsia. J Clin Gastroenterol. 2010;44(3):16772. doi: 10.1097/MCG.0b013e3181c64c69. 
2. Tack J, Talley NJ, Camilleri M. Functional gastroduodenal disorders. Gastroenterology 2006;130:1466-79. doi: 10.1053/j.gastro.2005.11.059.

3. Otero W, Gómez M, Otero L. Enfoque del paciente con dispepsia y dispepsia funcional. Rev Colomb Gastroenterol. 2014;29:132-8.

4. Talley NJ, Ford AC. Functional dyspepsia. N Engl J Med. 2015;373:1853-63. doi: 10.1056/NEJMra1501505.

5. Halder SLS, Talley NJ. Functional dyspepsia: A New Rome III Paradigm. Curr Treat Options Gastroenterol. 2007;10(4):259-72. doi: 10.1007/s11938-007-0069-0.

6. Talley NJ, Vakil NB, Moayyedi P. American gastroenterological association technical review on the evaluation of dyspepsia. Gastroenterology. 2005;129(5):1756-80. doi: 10.1053/j.gastro.2005.09.020.

7. Talley NJ, Vakil N. Practice Parameters Committee of the American College of Gastroenterology. Guidelines for the management of dyspepsia. Am J Gastroenterol. 2005;100(10):2324-37. doi: 10.1111/j.15720241.2005.00225.x.

8. Stanghellini V, Chan FK, Hasler WL, Malagelada JR, Suzuki $\mathrm{H}$, Tack J, et al. Gastroduodenal disorders. Gastroenterology. 2016;150:1380-92. doi: 10.1053/j.gastro.2016.02.011.

9. Delaney BC, Wilson S, Roalfe A, Roberts L, Redman V, Wearn A, et al. Cost effectiveness of initial endoscopy for dyspepsia in patients over age 50 years: a randomised controlled trial in primary care. Lancet. 2000;356(9246):1965-9.

10. Bytzer P. Diagnostic approach to dyspepsia. Best Pract Res Clin Gastroenterol. 2004;18:681-93. doi: 10.1016/j. bpg.2004.04.005.

11. Pineda LF, Otero W, Gómez M, Arbeláez V. Enfermedad estructural y valor predictivo de la Historia Clínica en pacientes con dispepsia no investigada. Rev Col Gastroenterol. 2004;19:13-25.

12. Gómez M, Otero W, Rincón J. Frecuencia de colelitiasis en dispepsia funcional, enfermedad por reflujo gastro-esofágico y en pacientes asintomáticos. Rev Colomb Gastroenterol. 2007;22(3):64-172.

13. Sugano K, Tack J, Kuipers EJ. Kyoto global consensus report on Helicobacter pylori gastritis. Gut. 2015;64:1353-67. doi: 10.1136/gutjnl-2015-309252.

14. Malfertheiner P, Megraud F, O’Morain CA, Gisbert JP, Kuipers EJ, Axon AT, et al. Management of Helicobacter pylori infection: The Maastricht V/Florence Consensus Report. Gut. 2017;66:6-30. doi: 10.1136/ gutjnl-2016-312288.

15. Bamber J, Cosgrove D, Dietrich CF, Fromageau J, Bojunga J, Calliada F, et al. EFSUMB guidelines and recommen- dations on the clinical use of ultrasound elastography. Part 1: Basic principles and technology. Ultraschall Med. 2013;34(2):169-84. doi: 10.1055/s-0033-1335205.

16. Pineda LF, Rosas MC, Amaya M, Rodríguez A, Luque A, Agudelo F, et al. Guía de Práctica Clínica para el diagnóstico y tratamiento de la dispepsia en adultos. Rev Colomb Gastroenterol. 2015;30(Suppl 1):9-16.

17. Godfrey EM, Rushbrook SM, Carroll NR. Endoscopic ultrasound: a review of current diagnostic and therapeutic applications. Postgrad Med J. 2010;16(4):111-22. doi: 10.1136/pgmj.2009.096065.

18. Yao K. Endoscopic diagnosis of early gastric cancer. Ann Gastroenterol. 2013;26(1):11-22.

19. Catalano MF, Sahai A, Levy M, Romagnuolo J, Wiersema $\mathrm{M}$, Brugge W, et al. EUS-based criteria for the diagnosis of chronic pancreatitis: the Rosemont classification. Gastrointest Endosc. 2009;69(7):1251-61. doi: 10.1016/j. gie.2008.07.043.

20. Russo MW, Wei JT, Thiny MT, Gangarosa LM, Brown A, Ringel Y, et al. Digestive and liver diseases statistics, 2004. Gastroenterology. 2004;126(5):1448-53. doi: 10.1053/j. gastro.2004.01.025.

21. Sakorafas GH, Milingos D, Peros G. Asymptomatic cholelithiasis: is cholecystectomy really needed? A critical reappraisal 15 year after the introduction of laparoscopic cholecystectomy. Dig Dis Sci. 2007,52:1313-25. doi: 10.1007/ s10620-006-9107-3.

22. Enck P, Azpiroz F, Boeckxstaens G, Elsenbruch S, FeinleBisset C, Holtmann G, et al. Functional dyspepsia. Nat Rev Dis Primers. 2017;3:17081. doi: 10.1038/nrdp.2017.81.

23. Sahai AV, Penman ID, Mishra G, Williams D, Pearson A, Wallace $\mathrm{MB}$, et al. An assessment of the potential value of endoscopic ultrasound as a cost-minimizing tool in dyspeptic patients with persistent symptoms. Endoscopy. 2001;33(8):662-7. doi: 10.1055/s-2001-16223.

24. Chang KJ, Erickson RA, Chak A, Lightdale C, Chen YK, Binmoeller KF, et al. EUS compared with endoscopy plus transabdominal US in the initial diagnostic evaluation of patients with upper abdominal pain. Gastrointest Endosc. 2010;72(5):967-74. doi: 10.1016/j.gie.2010.04.007.

25. Lee YT, Lai AC, Hui Y, Wu JC, Leung VK, Chan FK, et al. EUS in the management of uninvestigated dyspepsia. Gastrointest Endosc. 2002;56(6):842-8.

26. Studdert DM, Mello MM, Sage WM. Defensive medicine among high-risk specialist physicians in a volatile malpractice environment. JAMA. 2005;293:2609-261. doi: 10.1001/jama.293.21.2609. 